\title{
Relative Effects of Barrier Precautions and Topical Antibiotics on Nosocomial Bacterial Transmission: Results of Multi-compartment Models
}

\author{
B. Boldin ${ }^{\mathrm{a}, *}$, M.J.M. Bonten ${ }^{\mathrm{b}}$, O. Diekmann ${ }^{\mathrm{a}}$ \\ ${ }^{a}$ Department of Mathematics, University of Utrecht, Budapestlaan 6, 3584 CD Utrecht, \\ The Netherlands \\ ${ }^{b}$ Department of Internal Medicine, Division of Infectious Diseases, University Medical \\ Center Utrecht, Heidelberglaan 100, 3584 CX Utrecht, The Netherlands
}

Received: 5 December 2006 / Accepted: 22 February 2007 / Published online: 24 April 2007 (C) Society for Mathematical Biology 2007

\begin{abstract}
Nosocomial bacterial infections in critically ill patients are generally preceded by asymptomatic carriage (i.e. colonization) at one, or even several, body sites such as the skin, the gastro-intestinal and the respiratory tract. Different routes of transmission between the colonized sites create a complex epidemiology, which is additionally complicated by the smallness of the patient population size and the rapid patient turnover, characteristic for intensive care units (ICUs). Naturally occurring large fluctuations in the prevalence of colonization make it very difficult to determine the efficacy of control measures that aim to reduce the prevalence of antibiotic-resistant bacteria in ICUs. Theoretical models can sharpen our intuition through carefully designed thought experiments. In this spirit, we introduce and investigate two models that incorporate the fact that patients may be colonized at multiple body sites. Our study can be applied to several pathogens commonly found in ICUs, such Pseudomonas Aeruginosa, enteric Gram-negative bacteria, MRSA and enterococci. We evaluate the effects of barrier precautions (improved hygiene, use of gloves and gowns, etc.) and of administration of nonabsorbable antibiotics on the prevalence of colonization in ICUs and find that the effect of the controversial, though widely used, antibiotic prophylaxis can only be substantial if the patient-to-patient transmission has already been reduced to a subcritical level by barrier precautions. Taking into account that the very use of antibiotics may increase the selection for resistant strains and may thereby only add to the ever increasing problem of antibiotic resistance, our findings hence represent a firm theoretical argument against the routine use of topical antimicrobial prophylaxis for infection control.
\end{abstract}

Keywords Infection control - Intensive care unit - Basic reproduction ratio - SDD · MRSA · Antibiotic resistance $\cdot$ Selective decontamination of the digestive tract

\footnotetext{
*Corresponding author.

E-mail address: boldin@math.uu.nl (B. Boldin).
} 


\section{Introduction}

Infections with antibiotic-resistant microorganisms frequently occur in critically ill patients. In almost all cases, infection is preceded by asymptomatic carriage, i.e., colonization. Although different pathogens have different preferential colonization sites, several body sites may eventually become colonized, even those that are protected in healthy individuals. After one week of hospitalization, colonization of the respiratory tract, gastrointestinal tract and skin with potentially pathogenic microorganisms occurs in almost all critically ill patients (Bonten et al., 1996).

Intensive care units (ICUs) generally hold a small number of patients (typically 5-50), with an average length of stay that may very well be less than one week. Several routes of initiation of colonization can be distinguished. Patients may become colonized during their stay in the ICU through cross-transmission, a process that depends on the colonization pressure, i.e., the number/proportion of other patients already being colonized (Bonten et al., 1998). In some patients, susceptible microorganisms develop antibiotic resistance, either through specific mutations or through horizontal transfer of resistance genes. These events usually occur under antibiotic exposure and this mode of acquisition of resistance has been labeled endogenous acquisition of resistance. Finally, patients may be admitted to an ICU while being colonized, either at a level that is detectable by microbiological cultures, or in bacterial amounts that do not (yet) exceed the detection limits of sampling tests. In the latter case, selection through antibiotics may ultimately reveal 'new' cases of colonization. The smallness of ICU populations, rapid patient turnover and different routes of transmission create a complex epidemiology, with naturally occurring large fluctuations in the prevalence of colonization (Bonten et al., 2001).

Several infection control strategies can be used to reduce the prevalence of colonization in such a setting. Barrier precautions (e.g. hand disinfection, use of gloves and gowns, isolation of patients) aim to reduce cross-transmission (i.e. patient-to-patient transmission) and, although to a much smaller extent, endogenous transmission (transmission of microorganisms from one patient's body part to another, as yet uncolonized, part).

Administration of antibiotics, on the other hand, has many different facets. When administered intravenously or topically, antibiotics may serve as a way to reduce the prevalence of colonization in ICUs, either by reducing or by completely eradicating the susceptible flora, thereby also preventing the development of resistance (de Jonge et al., 2003; van Saene et al., 2003). Yet, the very use of antibiotics may also increase the selection pressure and the emergence of resistant strains and thereby only adding to the ever increasing problem of antibiotic resistance.

Enteral administration of nonabsorbable antibiotics, as done in selective decontamination of the digestive tract (SDD), is highly controversial: topical intestinal application of nonabsorbable antibiotics in ICU patients was part of a successful strategy to control an outbreak of multi-resistant Gram-negative bacteria in a French ICU (BrunBuisson et al., 1989), but was associated with increased resistance in some other ICUs (Lingnau et al., 1997; Verwaest et al., 1997). In all successful interventions, SDD was presumably instrumental in reducing the spread in the ICU (de Jonge et al., 2003; van Saene et al., 2003). However, in all such interventions barrier precautions and antibiotic use were introduced simultaneously, but the relative importance of the two control measures has never been determined. 
We hence developed a theoretical setting to investigate the relative effects of different interventions on the prevalence of colonization in ICUs. The small population sizes and rapid patient turnover that are typical for intensive care units, make it natural to formulate and study a stochastic model. But for precisely the same reasons, one needs to be very careful when drawing conclusions regarding the efficacy of different control measures. Namely, if we implement a certain intervention for some time and this intervention appears to be successful, to what extent is the reduction of colonization a result of random fluctuations and to what extent is it a result of the control measure? We therefore also formulated a deterministic counterpart of the stochastic model and compared the conclusions regarding the relative effects of different interventions with the ones drawn from the Markov chain model.

We present both models and the results in Sections 3 and 4. But to begin with, we now first describe the epidemiology and patient dynamics in intensive care units.

\section{The epidemiology and the dynamics in the ICU}

Our study does not focus on any pathogen in particular. The microorganism we have in mind is characterized by the following properties: it is a pathogen widely found in ICUs, it can colonize several sites of a patient's body (including the gastro-intestinal tract) and is resistant to some antibiotics, but susceptible to the intestinally administered nonabsorbable antibiotics. Pseudomonas aeruginosa, enteric Gram-negative bacteria, methicillin-resistant Staphylococcus aureus (MRSA) and enterococci are all examples of such a pathogen.

We shall, however, single out three body sites that can become colonized: the skin, the gut and the lungs, and label the patients in the ICU according to the parts of their bodies that are colonized at the time. Each of the body sites is assumed to be either colonized or not (in other words, we neglect the fact that individuals may differ in the bacterial load they carry at various sites) and so the label GS, for example, indicates that an individual is colonized in the gut and on the skin, but not (yet) in the lungs. In this way we can at any point in time characterize each patient in the ICU by one of the following eight labels: 0 , $G, S, L, G S, G L, S L, G S L$.

Skin colonization may lead to contamination of the health care workers' hands during patient care and is a prerequisite for cross-transmission. We shall use the term "skin colonization" throughout the paper and describe by this term any situation that may lead to cross-transmission (e.g. a contaminated respiratory tubing system or suffering from diarrhea). In fact, colonization of the lungs/intestines is characterized as one that cannot be transmitted through contaminated hands. It is, however, possible that a patient colonized in the lungs or in the gut transmits the bacteria to the skin by endogenous transmission.

The lungs, the gastro-intestinal tract and the skin are certainly sites frequently colonized by one or even several pathogens in ICUs (Bonten et al., 1996). But more importantly, these three sites capture precisely the three substantially different manners of colonization in view of the possible control measures: patients colonized on the skin are infectious and hence an immediate threat to other patients (and health care workers). We can reduce this threat by applying barrier precautions. Patients colonized in the gut/lungs are not yet infectious but may become so through endogenous transmission (e.g. developing diarrhea or having a respiratory tube inserted) and while reducing the chance of 
bacteria spreading from the gut to the skin can be achieved by barrier precautions as well as antibiotics, preventing the bacteria to spread from the lungs to the skin can only be done by improved hygiene. These three body sites hence capture the essence and any additional colonization site would mainly only complicate the notation as well as the computations without adding new qualitative aspects.

\subsection{Transmission}

We have already indicated in the previous subsection the possible transmission routes. Here we describe them again in order to also introduce the notation and some additional assumptions. We distinguish the following transmissions:

1. Cross-transmission, meaning that a patient colonized on the skin transmits the infection, via health care workers, to another patient's skin. All individuals not yet colonized on the skin are assumed to be equally susceptible to an infection (i.e. possible colonization of other body sites does not influence their susceptibility). Infectiousness of patients colonized on the skin is assumed to be independent of the colonization status of their other parts. We denote the (constant) rate at which an individual colonized (at least) on the skin infects type $0, G, L, G L$ by $\beta$.

2. Endogenous transmission, where a patient spreads the bacteria from one of the colonized sites to one of its as yet uncolonized parts. The following internal transmissions are possible:

- From the gut to the skin

- From the skin to the gut or to the lungs

- From the lungs (possibly via the respiratory tube) to the skin

The list of all possible endogenous transmissions and the (constant) rates at which they occur is as follows:

$$
\begin{array}{ll}
G \stackrel{\alpha_{G S}}{\longrightarrow} G S, & G L \stackrel{\alpha_{G S}}{\longrightarrow} G S L, \\
L \stackrel{\alpha_{L S}}{\longrightarrow} S L, & G L \stackrel{\alpha_{L S}}{\longrightarrow} G S L, \\
S \stackrel{\alpha_{S G}}{\longrightarrow} G S, & S L \stackrel{\alpha_{S G}}{\longrightarrow} G S L, \\
S \stackrel{\alpha_{S L}}{\longrightarrow} S L, & G S \stackrel{\alpha_{S L}}{\longrightarrow} G S L .
\end{array}
$$

\subsection{Admission of new patients}

Individuals are admitted to the ICU at a constant rate $\lambda$. Newly admitted patients can be colonized at any of the three body parts. We shall assume that the probabilities with which a newly admitted individual is of a certain type are constant in time (one can for example imagine that the prevalence of this pathogen in the community at large is stationary, and that the individuals admitted to the ICU are sampled randomly from the outside community) and we denote them by $p_{0}, p_{G}, p_{S}, p_{L}, p_{G S}, p_{G L}, p_{S L}$ and $p_{G S L}$ and require that

$$
p_{0}+p_{G}+p_{S}+p_{L}+p_{G S}+p_{G L}+p_{S L}+p_{G S L}=1 .
$$




\subsection{Discharge/death}

Individuals are removed from the ICU (either due to discharge or death) with constant probability per unit of time $\mu$, which is independent of the patient's colonization status. The numbers $\lambda$ and $\mu$ are related to each other via the size $N^{*}$ of the ICU as follows: $\lambda=\mu N^{*}$ (see Appendix A).

\section{The modeling}

The typically low number of patients in ICUs makes a stochastic model a more suitable and natural description of the process than a deterministic one. We shall nevertheless first formulate a deterministic model (for the reasons mentioned in the Introduction) and then later on compare some of its features to the results of the simulations of the stochastic version of the model.

\subsection{The deterministic model}

If the population sizes were large, we could describe the dynamics in the ICU by the following set of ordinary differential equations:

$$
\begin{aligned}
& \dot{x}_{0}=\mu p_{0}-(\sigma I+\mu) x_{0}, \\
& \dot{x}_{G}=\mu p_{G}-\left(\sigma I+\alpha_{G S}+\mu\right) x_{G}, \\
& \dot{x}_{S}=\mu p_{S}+\sigma I x_{0}-\left(\alpha_{S G}+\alpha_{S L}+\mu\right) x_{S}, \\
& \dot{x}_{L}=\mu p_{L}-\left(\sigma I+\alpha_{L S}+\mu\right) x_{L}, \\
& \dot{x}_{G S}=\mu p_{G S}+\left(\sigma I+\alpha_{G S}\right) x_{G}+\alpha_{S G} x_{S}-\left(\alpha_{S L}+\mu\right) x_{G S}, \\
& \dot{x}_{G L}=\mu p_{G L}-\left(\sigma I+\alpha_{G S}+\alpha_{L S}+\mu\right) x_{G L}, \\
& \dot{x}_{S L}=\mu p_{S L}+\left(\sigma I+\alpha_{L S}\right) x_{L}+\alpha_{S L} x_{S}-\left(\alpha_{S G}+\mu\right) x_{S L}, \\
& \dot{x}_{G S L}=\mu p_{G S L}+\left(\sigma I+\alpha_{L S}+\alpha_{G S}\right) x_{G L}+\alpha_{S L} x_{G S}+\alpha_{S G} x_{S L}-\mu x_{G S L} .
\end{aligned}
$$

Here, $x_{j}$ with $j \in\{0, G, S, L, G S, G L, S L, G S L\}$ represent the fractions of the corresponding subpopulations (indicated by the indices) and

$$
I=x_{S}+x_{G S}+x_{S L}+x_{G S L}
$$

denotes the fraction of individuals that are colonized on the skin and can hence (immediately) cause new cases of colonization. The new parameter $\sigma$ can be expressed in terms of the 'old' ones as $\sigma=\frac{\beta \lambda}{\mu}$.

The system (1) is a scaled version of the one written in terms of actual numbers (as opposed to fractions) and the interested reader can find all the details in Appendix A. For reader's convenience we present the possible changes in an individual's colonization status and the rates at which they occur also in the form of a diagram (see Fig. 1). 


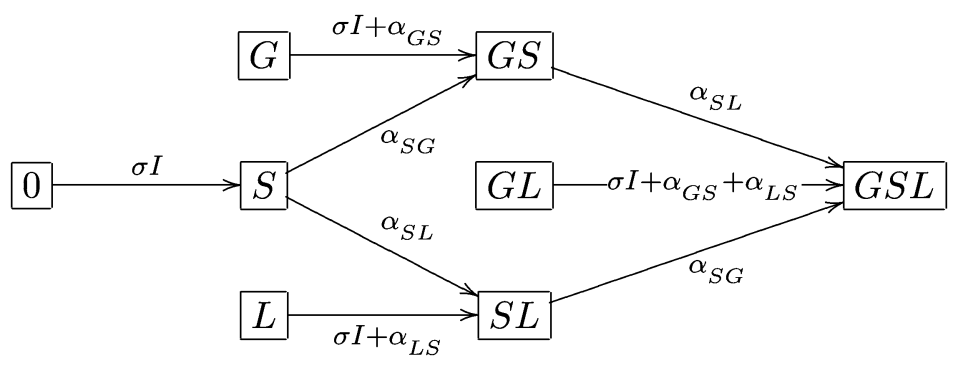

Fig. 1 Dynamics of an individual's colonization status.

\subsubsection{Equilibria and their stability}

In Appendix A we show that the equilibrium value of $I$ is obtained as a fixed point of the function $F$ given by

$$
\begin{aligned}
F(I)= & p+\frac{p_{0} \sigma I}{\sigma I+\mu}+\frac{p_{L}\left(\sigma I+\alpha_{L S}\right)}{\sigma I+\alpha_{L S}+\mu}+\frac{p_{G}\left(\sigma I+\alpha_{G S}\right)}{\sigma I+\alpha_{G S}+\mu} \\
& +\frac{p_{G L}\left(\sigma I+\alpha_{G S}+\alpha_{L S}\right)}{\sigma I+\alpha_{G S}+\alpha_{L S}+\mu} .
\end{aligned}
$$

where

$$
p=p_{S}+p_{G S}+p_{S L}+p_{G S L}
$$

When $F(0)>0$, the system (1) has a unique positive equilibrium and this equilibrium is locally asymptotically stable.

In the case $F(0)=0$ (for instance, when all newly admitted individuals are free of the infectious agent in question, i.e., $p_{0}=1$ ), the system admits a 'disease free' equilibrium, by which we mean an equilibrium consisting of only the subpopulations that cannot cause new infections. Whether the system also admits a nontrivial steady state, relies on the value of the basic reproduction ratio $\mathcal{R}_{0}$ (Diekmann and Heesterbeek, 2000), which is in the case $F(0)=0$ well defined and is equal to $\frac{\sigma}{\mu}$. Indeed, if, for instance, $p_{0}=1$ (see Appendix A for the derivation of $\mathcal{R}_{0}$ for other cases in which $F(0)=0$ ) and one skin infected individual is introduced into an otherwise infection free ICU, then this individual is expected to remain in the ICU for $\frac{1}{\mu}$ units of time and is in this time expected to infect the skin of $\frac{\sigma \cdot 1}{\mu}$ patients.

If $\mathcal{R}_{0}<1$ the infection free steady state is the only steady state and it is locally stable. When $\mathcal{R}_{0}>1$ there exists also a unique nontrivial equilibrium, which is then locally stable, while the infection free steady state is in that case unstable.

The proof of these claims is rather technical and can be found in Appendix A.

\subsection{The discrete time Markov chain model}

In addition to the deterministic model, we formulated a discrete time Markov chain model, which we investigated by way of simulations. The outcomes of simulations will be presented later on, here we only briefly describe the setting. 
In all the simulations, the reported ICU is assumed to hold 20 beds, all of which were assumed to be occupied at all times. The process was simulated as a discrete time process, taking one day as the unit of time. We have in fact simulated the process for a number of different choices of ICU size. We found that, even though the outcomes (say, colonization levels) are different, our main conclusions do not rely on the size of the intensive care unit.

The epidemiology (i.e. the colonization sites and the transmission routes) has already been described in Section 2. We now furthermore assume that the discharge and admission take place at one particular time each day. In this way, the only thing that changes in the ICU in the course of one day are the patients' colonization statuses.

The length of stay of each patient in the ICU is taken to be geometrically distributed with parameter $\mu$. After the discharge has taken place, the available beds are immediately filled with new patients. The colonization statuses (i.e. labels) of newly admitted patients are distributed according to the admission probabilities $p_{j}$. The endogenous transmissions are taken to be geometrically distributed with constant rates already introduced in Section 2. Patient-to-patient transmission, on the other hand, is a density dependent process. The cross-transmission rate is proportional to the number of patients in the ICU that are colonized on the skin and is given by

$$
\frac{\sigma \times \text { the number of patients colonized on the skin }}{\text { the number of patients in the ward }-1} \text {. }
$$

Since this rate may change in time, we update it on a daily basis, after each round of discharge and admission. Furthermore, transmissions are assumed to be independent of one another.

\section{Infection control measures}

Administration of topical, nonabsorbable antibiotics has no effect on the colonization of the skin or the lungs. It can, however, have two different effects on the individual's gastrointestinal tract: (i) it either reduces the rate of endogenous transmission of bacteria from the gut to the skin (although bacterial loads were not explicitly included, one can imagine that the lower rate is a result of the reduction in the bacterial load due to antibiotic therapy), but the patient remains colonized until discharge, or, (ii) colonization can be completely eradicated before the patient leaves the ICU. Both of these scenarios were investigated but since the outcomes were very similar we shall only present the results of the former scenario. Let us only remark that the latter requires a modification of both models to take into account the possibility that the intestinal carriage is successfully eliminated during patient's ICU stay.

Barrier precautions, on the other hand, can have an effect on several parameters. Primarily, they aim to reduce the cross-transmission parameter $\sigma$, but improved hygiene during patient care may also lower the rates $\alpha_{L S}$ and $\alpha_{G S}$. Furthermore, isolation of patients on admission or disinfection of a patient's skin on admission may lower the value of $p=p_{S}+p_{G S}+p_{S L}+p_{G S L}$.

In the deterministic setting we were in particular interested in the sensitivity of the equilibrium value of skin colonizations, $I^{*}$, to different parameters. The steady state value 
of $I$ is given implicitly as a fixed point of the function $F$ (see also Appendix A), given in (3).

The sensitivity of $I^{*}$ to a certain parameter, say $\theta$,

$$
S_{I^{*}, \theta}=\frac{\partial I^{*}}{\partial \theta}
$$

can be determined by using the implicit function theorem as

$$
S_{I^{*}, \theta}=\frac{\frac{\partial F}{\partial \theta}}{1-\frac{\partial F}{\partial I^{*}}},
$$

but since explicit computations lead to very cumbersome expressions that do not offer straightforward ways of comparing the sensitivities of $I^{*}$ to different parameters, we proceed with numerical examples.

We performed a series of numerical experiments for both models, aiming to determine the relative importance of different interventions. In particular, we tried to identify circumstances in which antibiotic therapy has a substantial effect and should be used to reduce the prevalence of colonization in an ICU. In the next section we present a couple of representative figures, along with the interpretation and the conclusions concerning implementation of control measures in ICUs. Some additional figures can be found in Appendix B.

\subsection{Results}

In reality some parameters, such as the discharge rate $\mu$ and the fraction of skin colonizations on admission $p$, are difficult to influence. We will therefore keep $\mu$ and $p$ constant in the examples presented in this section, taking the same values for the deterministic and the stochastic model: $\mu=0.1, p=0.2$. The reader can find some additional figures, in which the effects of reducing $p$ (which would correspond either to increased isolation on admission of patients that are colonized on the skin or to improved hygiene on admission) are displayed, in Appendix B (see Figs. B.2, B.3).

Studying the deterministic model, we found that the efficacy of topical antimicrobial prophylaxis relies heavily on (i) the level of cross-transmission in the ICU and (ii) the proportion of individuals that are colonized in the gut on admission. This can be seen from Fig. 2 in which three scenarios are presented; (a) low, (b) intermediate and (c) high level of intestinal colonization on admission:

(a) In Fig. 2a, the percentage of individuals that are colonized in the gut on admission is very low (15\%, including $5 \%$ of the total that are also already colonized on the skin). In this case, antibiotic prophylaxis may, of course, be beneficial on an individual level, but has virtually no effect on the endemic level of skin colonizations, regardless of the level of cross-transmission in the ICU.

(b) In Fig. 2b, the proportion of individuals that carry the pathogen in the gut on admission is $35 \%$, including $5 \%$ of the total that are also colonized on the skin. Here, reducing $\alpha_{G S}$ to zero has again almost no effect on $I^{*}$ when the level of cross transmission in the ICU is high (i.e. when $(1-p) \sigma / \mu>1$; see the top two lines), but when the cross-transmission rate is reduced so that on average the patients are not expected 


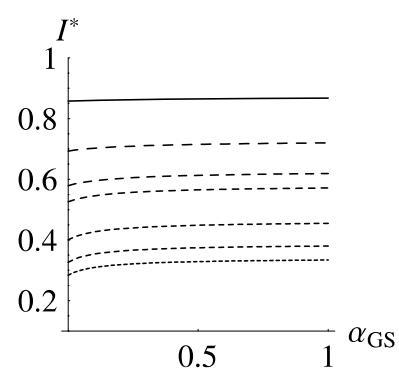

(A)

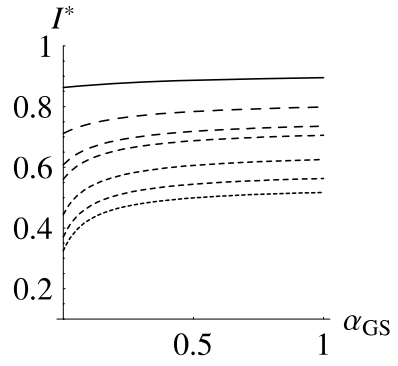

(B)

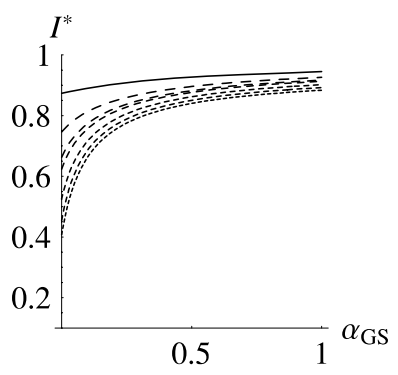

(C)

Fig. $2 I^{*}$ plotted as a function of $\alpha_{G S}$. In all three figures $\mu=\frac{1}{10}, \alpha_{L S}=\frac{1}{2}$ and, from the top to the bottom, $\sigma=\frac{1}{2}, \frac{1}{5}, \frac{1}{8}, \frac{1}{10}, \frac{1}{20}, \frac{1}{50}, 0$. In (a) $p_{j}=0.05$ for $j \neq 0$, (b) $p_{G}=0.2, p_{G L}=0.1, p_{j}=0.05$ for $j \neq\{0, G, G L\}$ and in (c) $p_{G}=0.5, p_{G L}=0.2, p_{j}=0.05$ for $j \neq\{G, G L\}$.

to transmit the infection to another patient during their stay in the ICU (the bottom lines), antibiotic prophylaxis starts to have an effect on $I^{*}$. In the case $\sigma=0$ (i.e. when there is no patient-to-patient transmission at all in the ward), antibiotic treatment has the capacity to reduce the prevalence of colonization by about a third (prevalence is reduced from 50\% to 35\%; see the bottom curve in Fig. 2b).

(c) Antibiotic prophylaxis can only really be beneficial on a population level when a large proportion of patients is amenable for treatment. However, even then, the level of cross-transmission in the ICU determines the efficacy of antibiotics. We observe from Fig. 2c that even though as much as $70 \%$ of the newly admitted patients are colonized in the gut on admission (and not yet on the skin), reducing $\alpha_{G S}$ to zero has almost no effect on $I^{*}$ when $(1-p) \sigma / \mu>1$ (see the top line in Fig. 2c; the prevalence is reduced for only about a fifth when $\alpha_{G S}$ is pushed to zero). Only when $(1-p) \sigma / \mu$ is brought below one (by applying barrier precautions), do antibiotics start to have an effect on the endemic level of skin colonizations and in such a case we can observe a two-fold reduction in $I^{*}$ (bottom three lines).

Figure 2 also demonstrates the sensitivity of $I^{*}$ to the cross-transmission parameter $\sigma$. We see from Figs. 2a, b that, while antibiotics have practically no effect on the endemic level of skin colonizations, barrier precautions that aim to reduce $\sigma$ have the potential to cause a two or even three-fold reduction in the prevalence: in Fig. 2a, the prevalence is reduced from $85 \%$ to $30 \%$, regardless of the value of $\alpha_{G S}$, while in Fig. $2 \mathrm{~b}$ the reduction is on average $45 \%$.

Only in the case when the proportion of individuals colonized in the gut on admission and the risk of transmitting the infection from the gut to the skin are high (Fig. 2c) can the antibiotics significantly reduce the prevalence of skin colonizations in the ICU. But, as mentioned before, the level of cross-transmission has to be reduced first. In such a case, therefore, a combination of the two control measures would bring the best results.

A similar experiment was performed taking $\alpha_{L S}=\frac{1}{15}$ (and so, while in the scenario depicted in Fig. 2 the patients are expected to transmit the bacteria from the lungs to the skin during their stay in the ICU, they are not expected to do so when $\alpha_{L S}=\frac{1}{15}$ ). The 


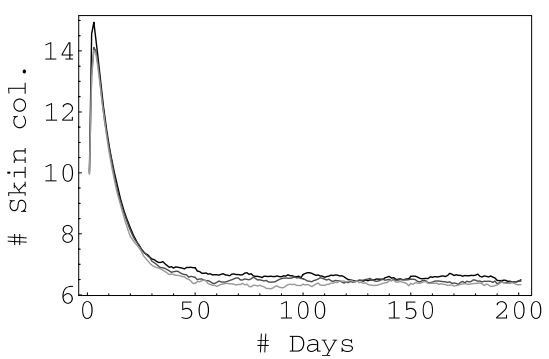

(A)

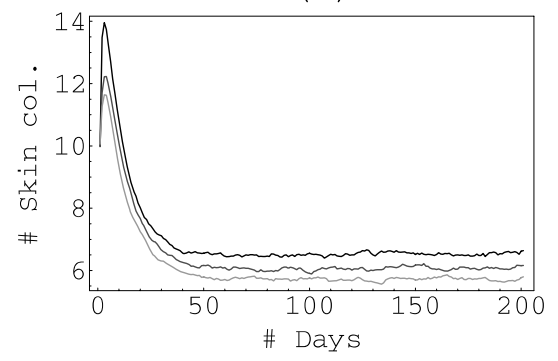

(C)

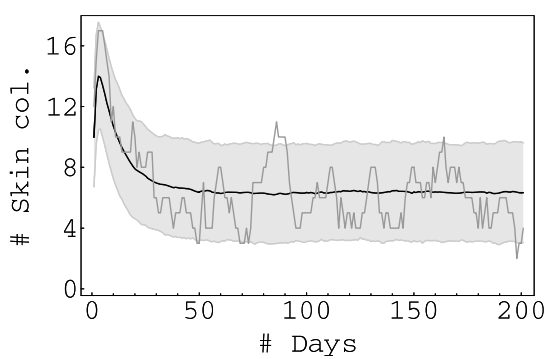

(B)

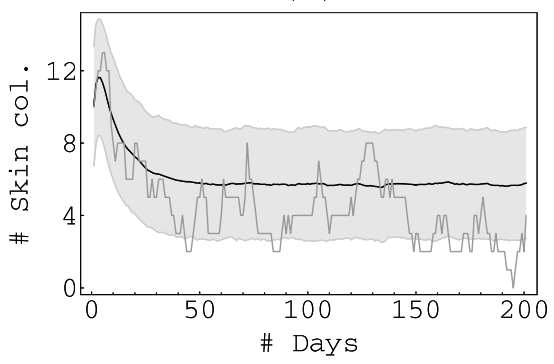

(D)

Fig. $3 p_{j}=0.05$ for $j \neq 0, \alpha_{S G}=0.5, \alpha_{L S}=0.5, \alpha_{S L}=0.2$. In (a) $\sigma=1$ and $\alpha_{G S}=1$ (black), $\alpha_{G S}=0.1$ (dark grey), $\alpha_{G S}=0.01$ (light grey). In (c) $\sigma=0.01$ and $\alpha_{G S}=1$ (black), $\alpha_{G S}=0.1$ (dark grey), $\alpha_{G S}=0.01$ (light grey). The right columns (figures (b) and (d)) show the mean of 1000 simulations, corresponding to $\alpha_{G S}=0.01$, along with the shaded area containing $90 \%$ of the simulations, and the result of a single simulation.

results are very similar to the ones in Fig. 2 and will not be shown here, but the interested reader can find them in Appendix B (Fig. B.1).

We now shift our attention to the results of the simulations. In all the figures presented here, the process was simulated 1000 times for a period of 200 days, starting with a randomly chosen initial condition. Just as in the deterministic case, we study three scenarios of intestinal colonization on admission: in Fig. 3 the proportion of individuals admitted with colonized gut is low, in Fig. 4 it is intermediate and it is high in Fig. 5.

The left columns (Figs. 3a, c, 4a, c and 5a, c) show the mean of 1000 simulations for three values of the endogenous rate $\alpha_{G S}$.

The right columns (Figs. 3b, d, 4b, d and 5b, d) contain the bottom curve of the left column (i.e. the one corresponding to the lowest $\alpha_{G S}$ ), the result of a single simulation and also a shaded grey area corresponding to the area containing $90 \%$ of all simulations.

The simulations support the conclusions obtained from the deterministic model: when the admission of intestinal colonizations to the ICU is low (Fig. 3), antibiotic prophylaxis has almost no effect on the mean level of the prevalence, regardless of the level of cross-transmission in the unit. This is also the case when the admission of intestinal colonizations is somewhat higher (see the top row in Fig. 4). However, lowering the rate $\alpha_{G S}$ does start to have an effect on the mean prevalence if the cross-transmission is reduced to almost zero (see Figs. 4a, c): while for $\sigma=1$ half of the patients in the unit are colonized 


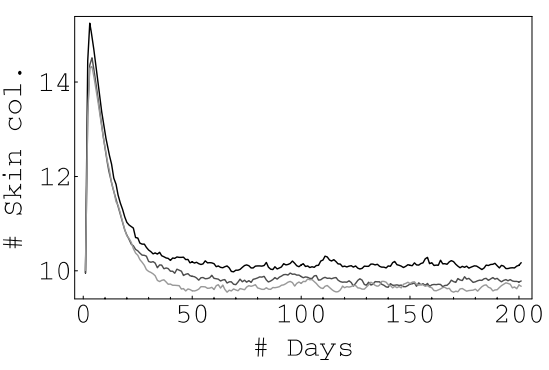

(A)

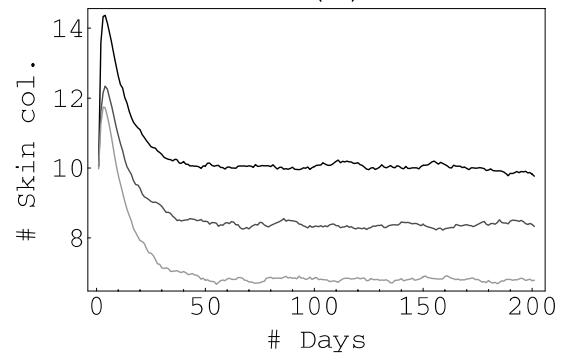

(C)

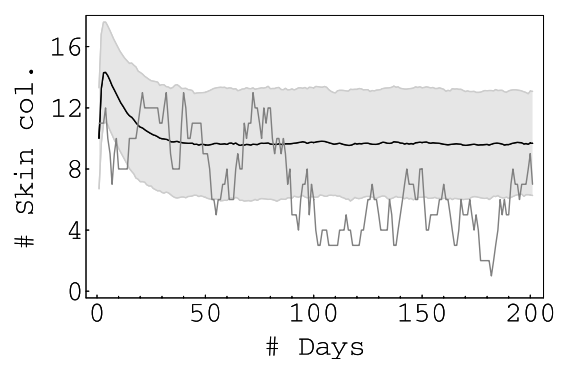

(B)

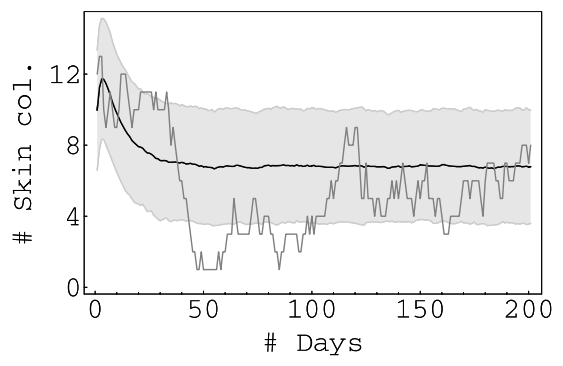

(D)

Fig. $4 p_{G}=0.2, p_{G L}=0.1, p_{j}=0.05$ for $j \neq 0, G, G L . \alpha_{S G}=0.5, \alpha_{L S}=0.5, \alpha_{S L}=0.2$. Top: $\sigma=1$, bottom $\sigma=0.01$. Left column: $\alpha_{G S}=1$ (black), $\alpha_{G S}=0.1$ (dark grey), $\alpha_{G S}=0.01$ (light grey). The right columns (figures (b) and (d)) show the mean of 1000 simulations, corresponding to $\alpha_{G S}=0.01$, along with the shaded area containing $90 \%$ of the simulations, and the result of a single simulation.

on average (regardless of the value of $\alpha_{G S}$ ), this number drops from 10 to 7 when $\sigma=0.01$ and we reduce $\alpha_{G S}$ from 1 to 0.01 . The grey area (that contains $90 \%$ of all simulations), shifts from $10 \pm 3$ (not shown) for $\alpha_{G S}=1$ to $7 \pm 3$ for $\alpha_{G S}=0.01$.

Figure 5 again shows that maintaining proper hygiene in the ICU is necessary if the antibiotics are to have an effect at all: in Fig. 5a, when the cross-transmission is high, antibiotics have no effect: only when $\sigma$ is considerably reduced do the antibiotics have an effect on the population level. This effect is now, due to the large proportion of newly admitted individuals with intestinal colonization, considerable: on average, the number of skin colonizations in the ICU is reduced from $17 \pm 3$ to $9 \pm 3$.

When focusing on the averages, therefore, the deterministic and the stochastic model echo the same message: antibiotic therapy as a means to reduce the prevalence of colonization in the ICU should only be used when a relatively high proportion of patients are colonized in the intestines on admission and furthermore the barrier precautions have already been applied to make sure that the occurrence of patient-to-patient transmission in the ICU is as low as possible. Otherwise, the benefits of antibiotic treatment may be high on an individual level, but the effect on the population level will be negligible.

Due to the low number of patients in ICUs and the rapid turnover, stochastic effects may lead to considerable fluctuations in the prevalence of skin colonization in the ICU. In $10 \%$ of the simulations that fall out of the grey area, we may observe long periods in which the prevalence remains below average, even decreases, for long periods of time before it 


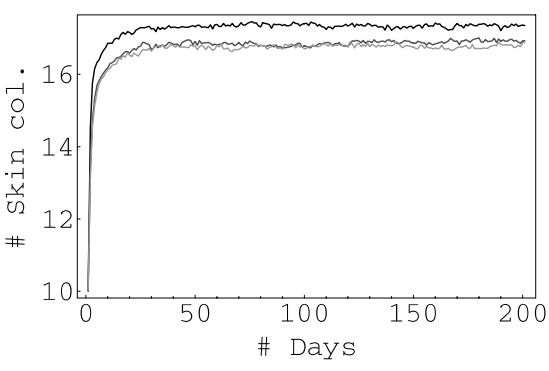

(A)

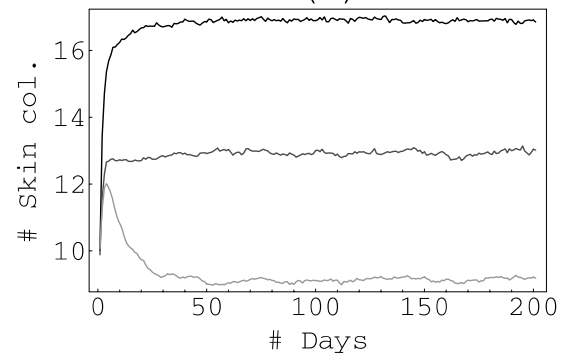

(C)

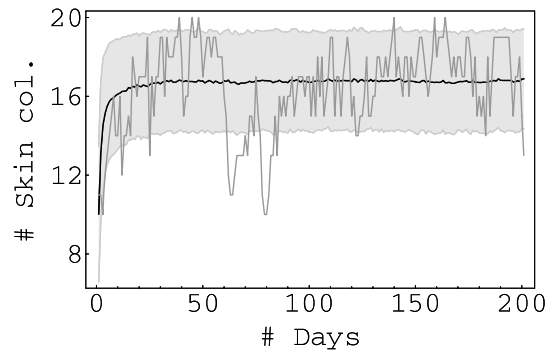

(B)

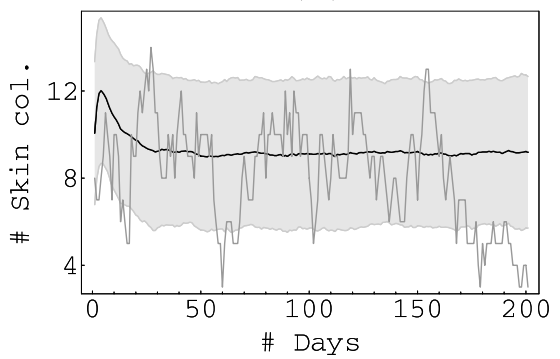

(D)

Fig. $5 p_{G}=0.5, p_{G L}=0.2, p_{j}=0.05$ for $j \neq 0, G, G L . \alpha_{S G}=0.5, \alpha_{L S}=0.5, \alpha_{S L}=0.2$. Top: $\sigma=1$, bottom $\sigma=0.01$. Left column: $\alpha_{G S}=1$ (black), $\alpha_{G S}=0.1$ (dark grey), $\alpha_{G S}=0.01$ (light grey). The right columns (figures (b) and (d)) show the mean of 1000 simulations, corresponding to $\alpha_{G S}=0.01$, along with the shaded area containing $90 \%$ of the simulations, and the result of a single simulation.

increases again. In observing single runs (or, in real life, one intervention in one ICU) one could, therefore, be tempted to believe that intestinal application of antibiotics has significant benefits even in the case of low/intermediate admission of intestinal colonization. The deterministic model and the figures representing the means of 1000 simulations show that this is not the case: if such an intervention seems to be successful in an ICU, it may very well be a matter of chance rather than due to real benefits of antibiotic prophylaxis.

\section{Discussion}

Complex pathogen dynamics, small patient populations and rapid patient turnover make the observation of the efficacy of different infection control measures in ICUs very difficult. Barrier precautions and intestinal administration of topical antibiotics are two measures that can be applied to reduce the prevalence of antibiotic resistant pathogens. Yet, prophylactic use of topical antibiotics is highly controversial (Bonten et al., 1996, 1998, 2001) as it may lead to increased occurrence of resistant strains. Moreover, different control measures are usually implemented simultaneously, precluding the evaluation of the effects of individual measures. To amend for the lack of theoretical background in this matter, we formulated a deterministic and a discrete time Markov chain model to describe the dynamics of antibiotic resistant bacteria in ICUs. We have demonstrated that intestinal 
application of nonabsorbable antibiotics, with the aim of reducing antibiotic resistance at the population level, can only be effective when (i) a high percentage of newly admitted patients is colonized in the intestines and (ii) cross-transmission rates in the ward are low. Our findings thus represent a firm theoretical argument against the routine use of topical antimicrobial prophylaxis for infection control.

Our study can be applied to several pathogens commonly found in ICUs, such as Pseudomonas aeruginosa, MRSA, enterococci and enteric Gram-negative bacteria. Simultaneous colonization at multiple body sites with such pathogens is universal among ICUpatients (Bonten et al., 1996). Pathogen dynamics, therefore, includes both the within-host and patient-to-patient transmission. From a conceptual point of view, these colonization sites can be divided in internal and external compartments, distinguished by the capacity to act as a source for cross-transmission. The model used in this study was based on this concept, with the skin as the representative for all body sites that can be contacted by health care workers, and thus be a source for cross-transmission. For the internal compartments we chose the lungs and the intestinal tract.

Under the vocal cords, the respiratory tract is usually sterile. Colonization frequently occurs in ICU-patients as the physiological defence mechanisms against aspirated microorganisms are breached because of intubation. Moreover, the commensal bacterial flora of the upper respiratory tract, susceptible to many antibiotics, rapidly disappears after ICU-admission, partly because of antibiotics. Subsequently, the upper and lower respiratory tract become colonized with the typical hospital-acquired bacterial flora, which is often resistant to many antibiotics (e.g. Pseudomonas aeruginosa, Enterobacteriaceae and MRSA). From the respiratory tract pathogens may reach the external compartment (where they can contaminate health care workers hands) during patient care. Eradication of bacterial colonization in the oropharyngeal cavity (part of the upper respiratory tract) can be achieved with topical antimicrobial agents (Bergmans et al., 2001). Yet, this approach does not completely eradicate colonization of the lower respiratory tract. In our analyses we therefore considered lung colonization as a constant source from where bacteria could reach the external compartment.

The intestinal tract is considered the most abundant source of pathogens causing hospital-acquired infections. As in the respiratory tract, the commensal bacterial flora that is susceptible to many antibiotics, is rapidly replaced after ICU-admission with the typical hospital-acquired bacterial flora. In contrast with the respiratory tract, however, eradication of this flora is achievable with nonabsorbable antibiotics.

Because of the constantly increasing prevalence of antibiotic resistance, prevention of transmission of such pathogens becomes more and more important. Barrier precautions are key in infection prevention in hospitals. In clinical practice, such measures include optimal hand hygiene after patient contact, placing a patient in a single-bed room and approaching the patient only when wearing gloves and gowns. Barrier precautions alone have, however, frequently been insufficient to prevent the spread of antibiotic resistant pathogens and additional preventive measures are needed, especially in ICUs.

Eradication of bacterial colonization could be a very effective control measure. For most antibiotic-resistant bacteria, colonization, once established, persists until patient discharge from the ICU. After that, reestablishment of the commensal flora occurs, possibly because of reduced antibiotic selective pressure. Several eradication strategies have been implemented, though frequently without success (Loeb et al., 2003). The concept of nonabsorbable antibiotics applied into the intestinal tract, usually through a nasogastric tube, 
has met considerable enthusiasm (van Saene et al., 2003). It has been tested frequently as part of SDD, in which intestinal decontamination is combined with oropharyngeal decontamination and a short course of intravenous antibiotic prophylaxis. The primary goal of SDD was to prevent the development of ICU-acquired respiratory tract infections. After more than forty randomized trials evaluating the effects of SDD, there is strong evidence that SDD indeed reduces incidences of ICU-acquired respiratory tract infections (van Saene et al., 2003). Moreover, ICU-survival among patients receiving SDD increased according to three recent studies (de Jonge et al., 2003; de la Cal et al., 2005; Krueger et al., 2002). The effects of SDD on the prevalence of antibiotic resistant bacteria, however, are controversial: SDD enhanced preexisting high antibiotic resistant prevalence levels (Lingnau et al., 1998), whereas SDD reduced such prevalence levels in units with low baseline prevalences (de Jonge et al., 2003). It is generally assumed that the intestinal component of SDD has the largest effect on increasing or decreasing unit-wide prevalence levels of antibiotic resistance. This assumption is supported by the reported effects of topical antimicrobial prophylaxis, as in SDD, as a measure for controlling outbreaks of antibiotic resistance (Brun-Buisson et al., 1989). Therefore, the use of SDD has been advocated as a routine measure to control antibiotic resistance in ICUs.

Our findings, to some extent, support this concept. Yet, optimal use of barrier precautions and a relatively high admission rate of intestinal carriage of resistant bacteria are prerequisites for the efficacy of intestinal decontamination. In most ICUs a considerable part of the patients is directly admitted from the community (such as trauma patients) and the admission prevalence of colonization with resistant bacteria will be low. To conclude, the results of this study, therefore militate against the routine use of topical antimicrobial prophylaxis for infection control.

\section{Appendix A The deterministic model revisited}

The aim of this part is to establish the number of equilibria of the system (1) and their stability. But first, we pay attention to the formulation of the model.

Let $N_{0}, N_{G}, N_{S}, N_{L}, N_{G S}, N_{G L}, N_{S L}, N_{G S L}$ denote the sizes of the subpopulations indicated by the indices. If the population sizes were large, we could describe the dynamics with the following system of ODEs:

$$
\begin{aligned}
\dot{N}_{0}= & \lambda p_{0}-\beta\left(N_{S}+N_{G S}+N_{S L}+N_{G S L}\right) N_{0}-\mu N_{0} \\
\dot{N}_{G}= & \lambda p_{G}-\beta\left(N_{S}+N_{G S}+N_{S L}+N_{G S L}\right) N_{G}-\left(\alpha_{G S}+\mu\right) N_{G}, \\
\dot{N}_{S}= & \lambda p_{S}+\beta\left(N_{S}+N_{G S}+N_{S L}+N_{G S L}\right) N_{0}-\left(\alpha_{S G}+\alpha_{S L}+\mu\right) N_{S}, \\
\dot{N}_{L}= & \lambda p_{L}-\beta\left(N_{S}+N_{G S}+N_{S L}+N_{G S L}\right) N_{L}-\left(\alpha_{L S}+\mu\right) N_{L}, \\
\dot{N}_{G S}= & \lambda p_{G S}+\beta\left(N_{S}+N_{G S}+N_{S L}+N_{G S L}\right) N_{G}-\left(\alpha_{S L}+\mu\right) N_{G S} \\
& +\alpha_{G S} N_{G}+\alpha_{S G} N_{S} \\
\dot{N}_{G L}= & \lambda p_{G L}-\beta\left(N_{S}+N_{G S}+N_{S L}+N_{G S L}\right) N_{G L}-\left(\alpha_{G S}+\alpha_{L S}+\mu\right) N_{G L}, \\
\dot{N}_{S L}= & \lambda p_{S L}+\beta\left(N_{S}+N_{G S}+N_{S L}+N_{G S L}\right) N_{L}-\left(\alpha_{S G}+\mu\right) N_{S L} \\
& +\alpha_{S L} N_{S}+\alpha_{L S} N_{L},
\end{aligned}
$$




$$
\begin{aligned}
\dot{N}_{G S L}= & \lambda p_{G S L}+\beta\left(N_{S}+N_{G S}+N_{S L}+N_{G S L}\right) N_{G L}+\left(\alpha_{L S}+\alpha_{G S}\right) N_{G L} \\
& +\alpha_{S L} N_{G S}+\alpha_{S G} N_{S L}-\mu N_{G S L} .
\end{aligned}
$$

The total population size,

$$
\begin{aligned}
N(t)= & N_{0}(t)+N_{G}(t)+N_{S}(t)+N_{L}(t)+N_{G S}(t)+N_{G L}(t)+N_{S L}(t) \\
& +N_{G S L}(t)
\end{aligned}
$$

changes according to the differential equation

$$
\dot{N}=\lambda-\mu N
$$

and so the equilibrium $N^{*}=\frac{\lambda}{\mu}$ is globally asymptotically stable. If we denote

$$
x_{j}=\frac{N_{j}}{N^{*}}, \quad j \in\{0, G, S, L, G S, G L, S L, G S L\},
$$

$I=x_{S}+x_{G S}+x_{L S}+x_{G S L}$ and $\sigma=\frac{\lambda \beta}{\mu}$ we can indeed rewrite the system as (1).

To study the steady states of (1), we first express

$$
x_{j}, \quad j \in\{0, G, S, L, G S, G L, S L, G S L\}
$$

in terms of $I^{*}$ (the steady state values will be indicated by a superscript *).

We have

$$
\left\{\begin{aligned}
x_{0}^{*} & =\frac{\mu p_{0}}{\sigma I^{*}+\mu}, \\
x_{G}^{*} & =\frac{\mu p_{G}}{\sigma I^{*}+\alpha_{G S}+\mu}, \\
x_{L}^{*} & =\frac{\mu p_{L}}{\sigma I^{*}+\alpha_{L S}+\mu}, \\
x_{G L}^{*} & =\frac{\mu p_{G L}}{\sigma I^{*}+\alpha_{G S}+\alpha_{L S}+\mu},
\end{aligned}\right.
$$

as the steady state values of four variables, and, using these, we can write the remaining four as

$$
\left\{\begin{array}{l}
x_{S}^{*}=\frac{\mu p_{S}}{\alpha_{S G}+\alpha_{S L}+\mu}+\frac{\sigma I^{*}}{\alpha_{S G}+\alpha_{S L}+\mu} x_{0}^{*}, \\
x_{G S}^{*}=\frac{\mu p_{G S}}{\alpha_{S L}+\mu}+\frac{\sigma I^{*}+\alpha_{G S}}{\alpha_{S L}+\mu} x_{G}^{*}+\frac{\alpha_{S G}}{\alpha_{S L}+\mu} x_{S}^{*}, \\
x_{S L}^{*}=\frac{\mu p_{S L}}{\alpha_{S G}+\mu}+\frac{\sigma I^{*}+\alpha_{L S}}{\alpha_{S G}+\mu} x_{L}^{*}+\frac{\alpha_{S L}}{\alpha_{S G}+\mu} x_{S}^{*}, \\
x_{G S L}^{*}=p_{G S L}+\frac{\sigma I^{*}+\alpha_{L S}+\alpha_{G S}}{\mu} x_{G L}^{*}+\frac{\alpha_{S L}}{\mu} x_{G S}^{*}+\frac{\alpha_{S G}}{\mu} x_{S L}^{*},
\end{array}\right.
$$

which are explicit expressions in terms of $I^{*}$ once we insert (A.1a). 
The steady state value $I^{*}$ satisfies

$$
I^{*}=x_{S}^{*}+x_{G S}^{*}+x_{S L}^{*}+x_{G S L}^{*}=: F\left(I^{*}\right),
$$

where $x_{S}^{*}, x_{G S}^{*}, x_{S L}^{*}$ and $x_{G S L}^{*}$ are given in (A.1). Hence, we need to investigate the fixed points of $F$.

After some manipulation we find that

$$
F(I)=p+\frac{p_{0} \sigma I}{\sigma I+\mu}+\frac{p_{L}\left(\sigma I+\alpha_{L S}\right)}{\sigma I+\alpha_{L S}+\mu}+\frac{p_{G}\left(\sigma I+\alpha_{G S}\right)}{\sigma I+\alpha_{G S}+\mu}+\frac{p_{G L}\left(\sigma I+\alpha_{G S}+\alpha_{L S}\right)}{\sigma I+\alpha_{G S}+\alpha_{L S}+\mu},
$$

where $p=p_{S}+p_{G S}+p_{S L}+p_{G S L}$ and the function $F$ has the following properties:

1. $F(0) \geq 0$

2. $F^{\prime}(I) \geq 0$ for $I \geq 0$

3. $\lim _{I \rightarrow \infty} F(I)=1$ and

4. $F^{\prime \prime}(I) \leq 0$ for $I \geq 0$

These properties ensure that, when $F(0)>0$ the function $F$ has a unique, strictly positive fixed point $I^{*}$. The system (1) then has a unique strictly positive steady state. Once $I^{*}$ is determined, we can calculate the steady state values of all state variables using (A.1).

Suppose now that $F(0)=0$. If we assume that $\alpha_{G S}>0$ and $\alpha_{L S}>0$ then $F(0)=0$ precisely when all newly introduced individuals are susceptible. In this case the value of $F^{\prime}(0)$ determines whether there will exist a nontrivial steady state. If $F^{\prime}(0)=\frac{\sigma}{\mu}=\mathcal{R}_{0}>1$ then we have a unique, strictly positive steady state and if $\mathcal{R}_{0} \leq 1$ then there is only the trivial steady state.

What remains is the possibility that at least one of $\alpha_{G S}, \alpha_{L S}$ equals zero. Let us only consider the case $\alpha_{G S}=0, \alpha_{L S}>0$ (the other two options are treated in the same way). Then $F(0)=0$ precisely when $p_{0}+p_{G}=1$, that is, all newly introduced patients are either not colonized at all or carry the bacteria in their gut. But since $\alpha_{G S}=0$ they can not spread the bacteria to their skin and we can treat individuals in $x_{0}, x_{G}$ as a heterogeneous susceptible population. Hence, $\mathcal{R}_{0}=p_{0} \frac{\sigma}{\mu}+p_{G} \frac{\sigma}{\mu}=\frac{\sigma}{\mu}=F^{\prime}(0)$ and an endemic equilibrium exists only when $\mathcal{R}_{0}>1$.

Let us now focus on the case when $F(0)>0$ and show that the (unique) equilibrium is locally asymptotically stable. The same line of reasoning can in fact also be applied to show the stability statements for the case $F(0)=0$.

To this end let

$$
x^{*}=\left(x_{0}^{*}, x_{G}^{*}, x_{S}^{*}, x_{L}^{*}, x_{G S}^{*}, x_{G L}^{*}, x_{S L}^{*}, x_{G S L}^{*}\right)
$$

denote the (unique) equilibrium of (1). The Jacobi matrix evaluated in $x^{*}$ is denoted by $J$ and has the following form 


$$
J=\left(\begin{array}{cccccccc}
J_{1} & 0 & -\sigma x_{0}^{*} & 0 & -\sigma x_{0}^{*} & 0 & -\sigma x_{0}^{*} & -\sigma x_{0}^{*} \\
0 & J_{2} & -\sigma x_{G}^{*} & 0 & -\sigma x_{G}^{*} & 0 & -\sigma x_{G}^{*} & -\sigma x_{G}^{*} \\
\sigma I^{*} & 0 & J_{3} & 0 & \sigma x_{0}^{*} & 0 & \sigma x_{0}^{*} & \sigma x_{0}^{*} \\
0 & 0 & -\sigma x_{L}^{*} & J_{4} & -\sigma x_{L}^{*} & 0 & -\sigma x_{L}^{*} & -\sigma x_{L}^{*} \\
0 & \sigma I^{*}+\alpha_{G S} & \sigma x_{G}^{*}+\alpha_{S G} & 0 & J_{5} & 0 & \sigma x_{G}^{*} & \sigma x_{G}^{*} \\
0 & 0 & -\sigma x_{G L}^{*} & 0 & -\sigma x_{G L}^{*} & J_{6} & -\sigma x_{G L}^{*} & -\sigma x_{G L}^{*} \\
0 & 0 & \sigma x_{L}^{*}+\alpha_{S L} & \sigma I^{*}+\alpha_{L S} & \sigma x_{L}^{*} & 0 & J_{7} & \sigma x_{L}^{*} \\
0 & 0 & \sigma x_{G L}^{*} & 0 & \sigma x_{G L}^{*}+\alpha_{S L} & \sigma I^{*}+\alpha_{L S}+\alpha_{G S} & \sigma x_{G L}^{*}+\alpha_{S G} & J_{8}
\end{array}\right),
$$

where

$$
\begin{aligned}
& J_{1}=-\left(\sigma I^{*}+\mu\right), \\
& J_{2}=-\left(\sigma I^{*}+\alpha_{G S}+\mu\right), \\
& J_{3}=\sigma x_{0}^{*}-\alpha_{S G}-\alpha_{S L}-\mu, \\
& J_{4}=-\left(\sigma I^{*}+\alpha_{L S}+\mu\right), \\
& J_{5}=\sigma x_{G}^{*}-\alpha_{S L}-\mu, \\
& J_{6}=-\left(\sigma I^{*}+\alpha_{G S}+\alpha_{L S}+\mu\right), \\
& J_{7}=\sigma x_{L}^{*}-\alpha_{S G}-\mu, \\
& J_{8}=\sigma x_{G L}^{*}-\mu .
\end{aligned}
$$

Let us define

$$
S=\left(\begin{array}{cccccccc}
1 & 0 & 0 & 0 & 0 & 0 & 0 & 0 \\
0 & 1 & 0 & 0 & 0 & 0 & 0 & 0 \\
0 & 0 & 1 & 0 & 0 & 0 & 0 & 0 \\
0 & 0 & 0 & 1 & 0 & 0 & 0 & 0 \\
0 & 0 & 0 & 0 & 1 & 0 & 0 & 0 \\
0 & 0 & 0 & 0 & 0 & 1 & 0 & 0 \\
0 & 0 & 0 & 0 & 0 & 0 & 1 & 0 \\
0 & 0 & -1 & 0 & -1 & 0 & -1 & 1
\end{array}\right)
$$

and calculate $K=S^{-1} J S$. We obtain

$$
K=\left(\begin{array}{cccccccc}
K_{1} & 0 & 0 & 0 & 0 & 0 & 0 & -\sigma x_{0}^{*} \\
0 & K_{2} & 0 & 0 & 0 & 0 & 0 & -\sigma x_{G}^{*} \\
\sigma I^{*} & 0 & K_{3} & 0 & 0 & 0 & 0 & \sigma x_{0}^{*} \\
0 & 0 & 0 & K_{4} & 0 & 0 & 0 & -\sigma x_{L}^{*} \\
0 & \sigma I^{*}+\alpha_{G S} & \alpha_{S G} & 0 & K_{5} & 0 & 0 & \sigma x_{G}^{*} \\
0 & 0 & 0 & 0 & 0 & K_{6} & 0 & -\sigma x_{G L}^{*} \\
0 & 0 & \alpha_{S L} & \sigma I^{*}+\alpha_{L S} & 0 & 0 & K_{7} & \sigma x_{L}^{*} \\
\sigma I^{*} & \sigma I^{*}+\alpha_{G S} & 0 & \sigma I^{*}+\alpha_{L S} & 0 & \sigma I^{*}+\alpha_{L S}+\alpha_{G S} & 0 & K_{8}
\end{array}\right),
$$


where

$$
K_{i}=J_{i} \quad \text { for } i=1,2,4,6
$$

and

$$
\begin{aligned}
& K_{3}=-\left(\alpha_{S G}+\alpha_{S L}+\mu\right), \\
& K_{5}=-\left(\alpha_{S L}+\mu\right), \\
& K_{7}=-\left(\alpha_{S G}+\mu\right), \\
& K_{8}=-\mu+\sigma\left(x_{0}^{*}+x_{G}^{*}+x_{L}^{*}+x_{G L}^{*}\right) .
\end{aligned}
$$

Now, it is clear from the form of $K$ that $K_{3}, K_{5}$ and $K_{7}$ are eigenvalues of $K$ (and therefore of $J$, since $K$ and $J$ are related by a similarity transformation) and from (A.4a-A.4c) that they are strictly negative. Our aim now is to show that all other eigenvalues of $K$ have negative real parts as well. In fact, we will see that all the eigenvalues of $K$ are real.

So let $\phi$ be an eigenvalue of $K$ and let $v^{T}=\left(v_{1}, \ldots, v_{8}\right)$ be a corresponding eigenvector. We have the following relations:

$$
\begin{aligned}
0= & \left(K_{1}-\phi\right) v_{1}-\sigma x_{0}^{*} v_{8}, \\
0= & \left(K_{2}-\phi\right) v_{2}-\sigma x_{G}^{*} v_{8}, \\
0= & \left(K_{4}-\phi\right) v_{4}-\sigma x_{L}^{*} v_{8}, \\
0= & \left(K_{6}-\phi\right) v_{6}-\sigma x_{G L}^{*} v_{8}, \\
0= & \sigma I^{*} v_{1}+\left(\sigma I^{*}+\alpha_{G S}\right) v_{2}+\left(\sigma I^{*}+\alpha_{L S}\right) v_{4} \\
& +\left(\sigma I^{*}+\alpha_{L S}+\alpha_{G S}\right) v_{6}+\left(K_{8}-\phi\right) v_{8}
\end{aligned}
$$

and

$$
\begin{aligned}
& 0=\sigma I^{*} v_{1}+\left(K_{3}-\phi\right) v_{3}+\sigma x_{0}^{*} v_{8}, \\
& 0=\left(\sigma I^{*}+\alpha_{G S}\right) v_{2}+\alpha_{S G} v_{3}+\left(K_{5}-\phi\right) v_{5}+\sigma x_{G}^{*} v_{8}, \\
& 0=\alpha_{S L} v_{3}+\left(\sigma I^{*}+\alpha_{L S}\right) v_{4}+\left(K_{7}-\phi\right) v_{7}+\sigma x_{L}^{*} v_{8} .
\end{aligned}
$$

We first consider the following situation:

CASE 1. $\alpha_{G S}>0, \alpha_{L S}>0, \alpha_{G S} \neq \alpha_{L S}$

Let us first see that in this case the spectrum of $K$ cannot contain $K_{i}$ for $i \in\{1,2,4,6\}$.

We show this by contradiction. Suppose that $\phi=K_{1}$. Since $x_{0}^{*} \neq 0, K_{1} \neq K_{2}, K_{4}$, $K_{6}$ and $I^{*} \neq 0$ we see from (A.5a-A.5e) that $v_{1}=v_{2}=v_{4}=v_{6}=v_{8}=0$ and then from (A.5f-A.5h) that also $v_{3}=v_{5}=v_{7}$ and so $v=0$, which cannot be the case since $v$ is an eigenvector.

In the same way we can see that neither of $K_{2}, K_{4}, K_{6}$ lies in the spectrum of $K$. We can hence use (A.5a-A.5d) to express $v_{1}, v_{2}, v_{4}, v_{6}$ in terms of $v_{8}$. We then obtain from 
(A.5e) that $v_{8}=0$ or

$$
\begin{gathered}
\frac{\sigma^{2} I^{*} x_{0}^{*}}{K_{1}-\phi}+\frac{\sigma\left(\sigma I^{*}+\alpha_{G S}\right) x_{G}^{*}}{K_{2}-\phi}+\frac{\sigma\left(\sigma I^{*}+\alpha_{L S}\right) x_{L}^{*}}{K_{4}-\phi} \\
+\frac{\sigma\left(\sigma I^{*}+\alpha_{G S}+\alpha_{L S}\right) x_{G L}^{*}}{K_{6}-\phi}+K_{8}-\phi=0 .
\end{gathered}
$$

Let us first assume that $v_{8} \neq 0$. Then, using (A.2a), (A.2b), (A.2d), (A.2f), (A.3) and (A.4d) we can rewrite (A.6) as

$$
(\phi+\mu)\left(\frac{\sigma x_{0}^{*}}{-K_{1}+\phi}+\frac{\sigma x_{G}^{*}}{-K_{2}+\phi}+\frac{\sigma x_{L}^{*}}{-K_{4}+\phi}+\frac{\sigma x_{G L}^{*}}{-K_{6}+\phi}\right)-(\phi+\mu)=0 .
$$

Hence, either $\phi=-\mu<0$, or

$$
\frac{\sigma x_{0}^{*} I^{*}}{-K_{1}+\phi}+\frac{\sigma x_{G}^{*} I^{*}}{-K_{2}+\phi}+\frac{\sigma x_{L}^{*} I^{*}}{-K_{4}+\phi}+\frac{\sigma x_{G L}^{*} I^{*}}{-K_{6}+\phi}-I^{*}=0 .
$$

Let us now write $\phi$ in (A.8) as $\phi=v+i \kappa$. Separating the real and the imaginary component, we see that necessarily $\kappa=0$ or

$$
\begin{aligned}
& \frac{\sigma x_{0}^{*}}{\left(-K_{1}+v\right)^{2}+\kappa^{2}}+\frac{\sigma x_{G}^{*}}{\left(-K_{2}+v\right)^{2}+\kappa^{2}}+\frac{\sigma x_{L}^{*}}{\left(-K_{4}+v\right)^{2}+\kappa^{2}} \\
& +\frac{\sigma x_{G L}^{*}}{\left(-K_{6}+v\right)^{2}+\kappa^{2}}=0,
\end{aligned}
$$

and since the steady state values of $x_{0}^{*}, x_{G}^{*}, x_{L}^{*}, x_{G L}^{*}$ are positive we conclude that $\kappa=0$.

Suppose now that $v=\phi \geq 0$. We can then estimate the left hand side of (A.8) as follows

$$
\begin{aligned}
\cdots< & \frac{\sigma x_{0}^{*} I^{*}}{-K_{1}}+\frac{\sigma x_{G}^{*} I^{*}}{-K_{2}}+\frac{\sigma x_{L}^{*} I^{*}}{-K_{4}}+\frac{\sigma x_{G L}^{*} I^{*}}{-K_{6}}-I^{*} \\
< & \frac{\sigma p_{0} I^{*}}{-K_{1}}+\frac{\sigma p_{G}^{*} I^{*}}{-K_{2}}+\frac{\sigma p_{L} I^{*}}{-K_{4}}+\frac{\sigma p_{G L} I^{*}}{-K_{6}}-I^{*} \\
< & p+\frac{p_{0} \sigma I^{*}}{-K_{1}}+\frac{p_{G}\left(\sigma I^{*}+\alpha_{G S}\right)}{-K_{2}}+\frac{p_{L}\left(\sigma I^{*}+\alpha_{L S}\right)}{-K_{4}} \\
& +\frac{p_{G L}\left(\sigma I^{*}+\alpha_{G S}+\alpha_{L S}\right)}{-K_{6}}-I^{*} \\
= & F\left(I^{*}\right)-I^{*}=0,
\end{aligned}
$$

where we have used in the second line that $x_{j}^{*}<p_{j}$ for $j \in\{0, G, L, G L\}$ and in the last that $I^{*}$ is a fixed point of $F$.

Hence, in order for $\phi$ to be a solution of (A.8), $\phi$ has to be negative.

Now, all that remains to consider in this first case are the eigenvalues of $K$ for which the corresponding eigenvector is such that $v_{8}=0$. Since we have already established that 
$\phi \neq K_{1}, K_{2}, K_{4}, K_{6}$ we obtain from (A.5a-A.5d) that also $v_{1}=v_{2}=v_{4}=v_{6}=0$. We can then rewrite (A.5f-A.5h) as

$$
\begin{aligned}
& 0=\left(K_{3}-\phi\right) v_{3}, \\
& 0=\alpha_{S G} v_{3}+\left(K_{5}-\phi\right) v_{5}, \\
& 0=\alpha_{S L} v_{3}+\left(K_{7}-\phi\right) v_{7}
\end{aligned}
$$

and observe that, since not all three of $v_{3}, v_{5}, v_{7}$ can be zero, the remaining three eigenvalues $K$ are $K_{3}, K_{5}$ and $K_{7}$ (as we have already observed in the beginning).

Since $K_{3}, K_{5}$ and $K_{7}$ are all strictly negative we have now shown that in the case when $\alpha_{G S}>0, \alpha_{L S}>0$ and $\alpha_{G S} \neq \alpha_{L S}$ all the eigenvalues of $K$ are real and strictly negative and so the steady state $x^{*}$ is locally asymptotically stable.

To complete the proof, we need to check what happens when the assumptions of the Case 1 are not met. This can happen in the following ways:

CASE 2. $\alpha_{G S}>0, \alpha_{L S}>0, \alpha_{G S}=\alpha_{L S}$

CASE 3. $\alpha_{G S}=0, \alpha_{L S}>0$

CASE 4. $\alpha_{G S}>0, \alpha_{L S}=0$

CASE 5. $\alpha_{G S}=0, \alpha_{L S}=0$

Since all these cases are handled in a similar manner, we shall give details for only one of them, namely, Case 2.

In the same way as before, we observe that $K_{1}$ and $K_{6}$ cannot be in the spectrum of $K$. In this case, however $K_{2}\left(=K_{4}\right)$ can be an eigenvalue and we will see that this necessarily is the case.

Let us first consider an eigenvalue of $K$, say $\phi$, such that $\phi \neq K_{2}$. We can then express $v_{1}, v_{2}, v_{4}$ and $v_{6}$ in terms of $v_{8}$ and just as in Case 1 obtain that $v_{8}=0, \phi=-\mu$ or that $\phi$ satisfies (A.8). Since the conclusion from Case 1, that in the latter case necessarily $\phi<0$, relied nowhere on the fact that $\alpha_{G S} \neq \alpha_{L S}$ we can carry it over right away to Case 2. Note however that, since $K_{2}=K_{4}$, we obtain a polynomial of degree 3 and hence three (strictly negative) roots. The 'missing' eigenvalue can only be equal to $K_{2}$, which is strictly negative and so we again conclude that $x^{*}$ is locally asymptotically stable.

This completes the proof.

\section{Appendix B Additional figures}

In Fig. B.1, we show the results of the same experiment as described in Section 4.1, only this time with $\alpha_{L S}=\frac{1}{15}$.

Figures B. 2 and B. 3 show the effects of reducing the proportion of individuals that are colonized on the skin already on admission. We begin at the right end point of the $p$-axis, with a certain starting distribution $\left\{p_{j}\right\}, j \in\{0, G, S, L, G S, G L, S L, G S L\}$. Reductions in $p=p_{S}+p_{G S}+p_{S L}+p_{G S L}$ are uniform in the four compartments $S, G S, S L, G S L$ and yield a uniform increase in $0, G, L, G L$. 


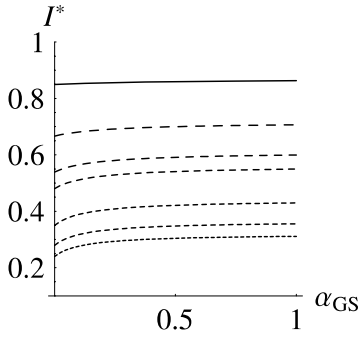

(A)

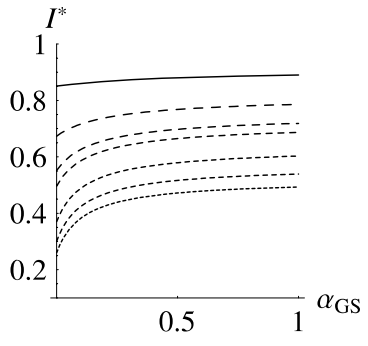

(B)

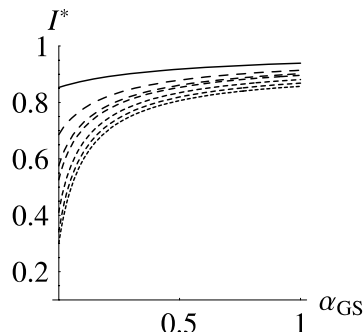

(C)

Fig. B.1 The endemic level of skin colonizations plotted as a function of $\alpha_{G S}$. In all three figures $\mu=\frac{1}{10}$, $\alpha_{L S}=\frac{1}{15}$ and, from the top to the bottom, $\sigma=\frac{1}{2}, \frac{1}{5}, \frac{1}{8}, \frac{1}{10}, \frac{1}{20}, \frac{1}{50}, 0$. In (a) $p_{j}=0.05$ for $j \neq 0$, (b) $p_{G}=0.2, p_{G L}=0.1, p_{j}=0.05$ for $j \neq\{0, G, G L\}$ and in (c) $p_{G}=0.5, p_{G L}=0.2, p_{j}=0.05$ for $j \neq\{G, G L\}$.

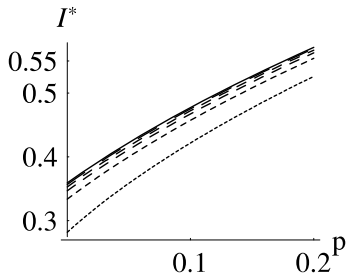

(A)

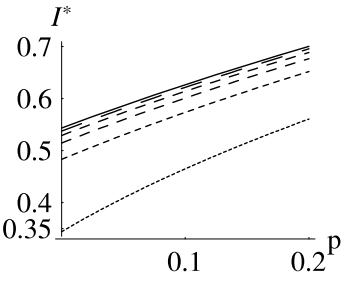

(B)

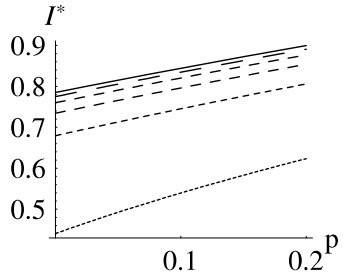

(C)

Fig. B.2 In all three figures $\sigma=0.1$ and, from top to bottom: $\alpha_{G S}=1,0.8,0.6,0.4,0.2,0$. The starting probabilities at the right end point of the p-axis are (a) $p_{j}=0.05$ for $j \neq 0$, (b) $p_{G}=0.2, p_{G L}=0.1$, $p_{j}=0.05$ for $j \neq\{0, G, G L\}$ and in (c) $p_{G}=0.5, p_{G L}=0.2, p_{j}=0.05$ for $j \neq\{G, G L\}$.

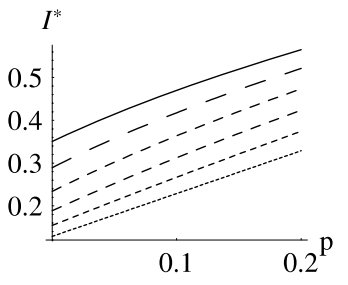

(A)

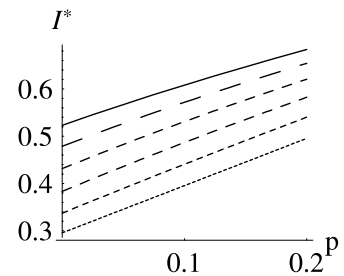

(B)

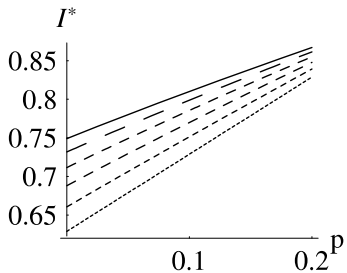

(C)

Fig. B.3 In all three figures $\alpha_{G S}=0.5$ and, from top to bottom: $\sigma=0.1,0.08,0.06,0.04,0.02,0$. The starting probabilities at the right end point of the p-axis are (a) $p_{j}=0.05$ for $j \neq 0$, (b) $p_{G}=0.2$, $p_{G L}=0.1, p_{j}=0.05$ for $j \neq\{0, G, G L\}$ and in (c) $p_{G}=0.5, p_{G L}=0.2, p_{j}=0.05$ for $j \neq\{G, G L\}$. 


\section{References}

Bergmans, D.C., Bonten, M.J.M., Gaillard, C.A., Palling, J.C., van der Geest, S., van Tiel, F.H., Beysens, A.J., de Leeuw, P.W., Stobberingh, E.E., 2001. Prevention of ventilator-associated pneumonia by oral decontamination: a prospective, randomized, double-blind, placebo-controlled study. Am. J. Respir. Crit. Care Med. 163(3), 382-388.

Bonten, M.J.M., Bergmans, D.C., Ambergen, A.W., de Leeuw, P.W., van der Geest, S., Stobberingh, E.E., Gaillard, C.A., 1996. Risk factors for pneumonia, and colonization of respiratory tract and stomach in mechanically ventilated ICU patients. Am. J. Respir. Crit. Care Med. 154, 1339-1346.

Bonten, M.J.M., Slaughter, S., Ambergen, A.W., Hayden, M.K., van Voorhis, J., Nathan, C., Weinstein, R.A., 1998. The role of "colonization pressure" in the spread of vancomycin-resistant enterococci: an important infection control variable. Arch. Intern. Med. 158(10), 1127-1132.

Bonten, M.J.M., Austin, D.J., Lipsitch, M., 2001. Understanding the spread of antibiotic resistant pathogens in hospitals: mathematical models as tools for control. Clin. Infect. Dis. 33, 1739-1746.

Brun-Buisson, C., Legrand, P., Rauss, A., Richard, C., Montravers, F., Besbes, M., Meakins, J.L., Soussy, C.J., Lemaire, F., 1989. Intestinal decontamination for control of nosocomial multiresistant gramnegative bacilli. Study of an outbreak in an intensive care unit. Ann. Intern. Med. 110(11), 873-881.

de Jonge, E., Schultz, M., Spanjaard, L., Bossuyt, P.P.M., Vroom, M.B., Dankert, J., Keseciglou, J., 2003. Effects of selective decontamination of digestive tract on mortality and acquisition of resistant bacteria in intensive care: a randomised controlled trial. Lancet 362(9389), 1011-1016.

de la Cal, M.A., Cerda, E., Garcia-Hierro, P., van Saene, H.K., Gomez-Santos, D., Negro, E., Lorente, J.A., 2005. Survival benefit in critically ill burned patients receiving selective decontamination of the digestive tract: a randomized, placebo-controlled, double-blind trial. Ann. Surg. 241(3), 424-430.

Diekmann, O., Heesterbeek, J.A.P., 2000. Mathematical Epidemiology of Infectious Diseases. Wiley Series in Mathematical and Computational Biology. Wiley, Chichester. Model building, analysis and interpretation.

Krueger, W.A., Lenhart, F.P., Neeser, G., Ruckdeshel, G., Schreckhase, H., Eissner, H.J., Forst, H., Eckart, J., Peter, K., Unertl, K.E., 2002. Influence of combined intravenous and topical antibiotic prophylaxis on the incidence of infections, organ dysfunctions, and mortality in critically ill surgical patients: a prospective, stratified, randomized, double-blind, placebo-controlled clinical trial. Am. J. Respir. Crit. Care Med. 166(8), 1029-1037.

Lingnau, W., Berger, J., Javorsky, F., Lejeune, P., Mutz, N., Benzer, H., 1997. Selective intestinal decontamination in multiple trauma patients: prospective, controlled trial. J. Trauma 42(4), 687-694.

Lingnau, W., Berger, J., Javorsky, F., Fille, M., Allerberger, F., Benzer, H., 1998. Changing bacterial ecology during a five-year period of selective intestinal decontamination. J. Hosp. Infect. 39(3), 195-206.

Loeb, M., Main, C., Walker-Dilks, C., Eady, A., 2003. Antimicrobial drugs for treating methicillin-resistant staphylococcus aureus colonization. Cochrane Database Syst. Rev. 4, CD003340.

van Saene, H.K., Petros, A.J., Ramsay, G., Baxby, D., 2003. All great truths are iconoclastic: selective decontamination of the digestive tract moves from heresy to level 1 truth. Intensive Care Med. 29(5), $677-690$.

Verwaest, C., Verhaegen, J., Ferdinande, P., Schetz, M., van der Berghe, G., Lauwers, P., 1997. Randomized, controlled trial of selective digestive decontamination in 600 mechanically ventilated patients in a multidisciplinary intensive care unit. Crit. Care Med. 25(1), 63-71. 